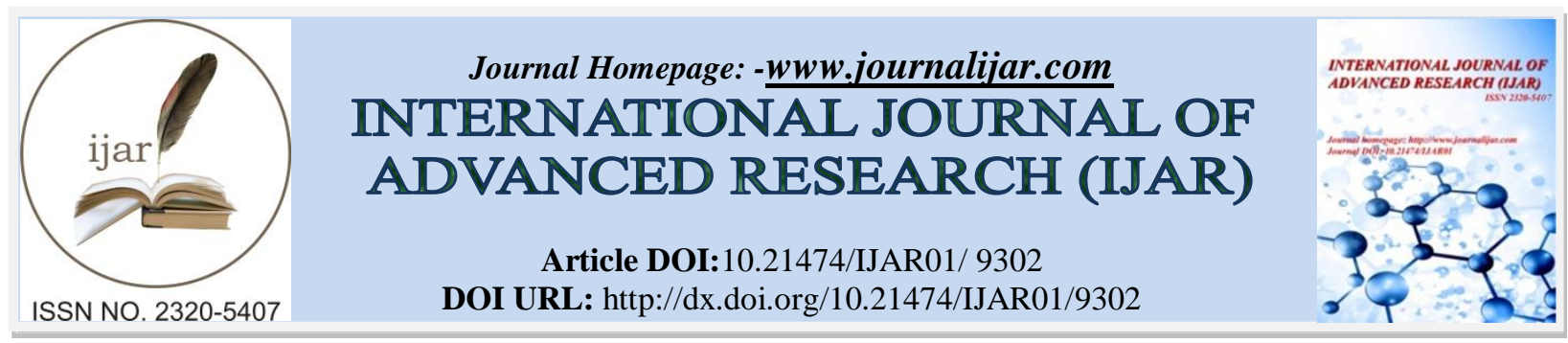

RESEARCH ARTICLE

\title{
MALIGNANT MIXED MULLERIAN TUMOR (UTERINE CARCINOSARCOMA) PRESENTED AS FIBROID UTERUS -A CASE REPORT.
}

\author{
Dr. Laxmi Aheer ${ }^{1}$, Dr. Taruna chaudhary ${ }^{2}$,Dr. Yogi Raj Joshi ${ }^{3}$ and Dr. Shilpa Agrawal ${ }^{4}$. \\ 1. Senior Demonstrator, Department of pathology. \\ 2. Assistant Professor, Department of Pathology. \\ 3. Associate Professor, Department of Pathology. \\ 4. Dr. Sampurnanand Medical College Jodhpur (Raj).
}

\section{Manuscript Info}

.........................

Manuscript History

Received: 15 April 2019

Final Accepted: 17 May 2019

Published: June 2019

\section{Abstract}

Malignant mixed mullerian tumor of the uterus, also called carcinosarcoma, is a very rare and aggressive type of malignancy with epithelial and mesenchymal component. It can occur anywhere along the female genital tract, like uterus, ovary, fallopian tube, cervix. Uterus is the commonest site, representing less than $5 \%$ of uterine malignancy. Here we presenting a case of $65 \mathrm{yr}$ old postmenopausal women admitted to hospital with complain of vaginal discharge from 2 month duration. Hysterectomy with bilateral salpingo-oopherectomy remain the mainstay of treatment, high rate of recurrence and metastasis suggest a need for lymphadenectomy and post operative adjuvant treatment.

Copy Right, IJAR, 2019,. All rights reserved.

\section{Introduction:-}

Malignant mixed mullerian tumor (MMMT) of the uterus is a very rare and aggressive type of malignancy with epithelial and mesenchymal components. Also called carcinosarcoma, metaplastic carcinoma and malignant mesodermal mixed tumor ${ }^{[1]}$. Sarcomatoid carcinoma is considered a different entity. Predominantly this tumor is found in postmenopausal women with uterine bleeding, pelvic pain and uterine enlargement. Usual site is uterus, ovary, fallopian tube, cervix, vagina, peritoneum, and extra genital site ${ }^{[2,3]}$. It has association with chronic estrogenic stimulation, radiation therapy, nulliparity, diabetes and obesity ${ }^{[4,5]}$.

\section{Case history}

65 yr old postmenopausal women admitted to hospital with complain of white vaginal discharge from 2 month duration only. No history of vaginal bleeding or abdomen pain. Previous history of D\&C showed inflammatory pathology with no evidence of malignancy. USG report showed bulky enlarged uterus measuring $11 \times 8.5 \times 7.7 \mathrm{~cm}$, heterogenous nodularity with area of calcification and increased vascularity all over uterus with indistinct endometrium with impressionof ?calcified fibroid / Adenomyosis. Preoperatively limited investigation were done and patient was posted for surgery. Total abdominal hysterectomy with bilateral salpingo-oopherectomy was done and specimen was sent to histopathological examination. No metastasis found in postoperative CECT abdomen.

Corresponding Author: - Dr. Yogi Raj Joshi.

Address:-Senior Demonstrator, Department of pathology. 
Postoperatively we received hysterectomy specimen with attached bilateral ovary and fallopian tube. Specimen was fixed overnight in $10 \%$ formalin and histopathological processing was started.

\section{Gross examination}

Uterus cervix with bilateral adnexa. Cervix was unremarkable. Endometrial canal dilated filled with polypoidal mass measuring $8 \times 6 \times 5 \mathrm{~cm}$. On serial sectioning cut surface of mass was grey white variegated with areas of calcification and necrosis. Tumor had gritty sensation while cutting. Mass was attached to the anterior wall of body of uterus and extending into myometrium. Mass was extending into the cervical canal. Both ovary and fallopian tubes are unremarkable grossly.

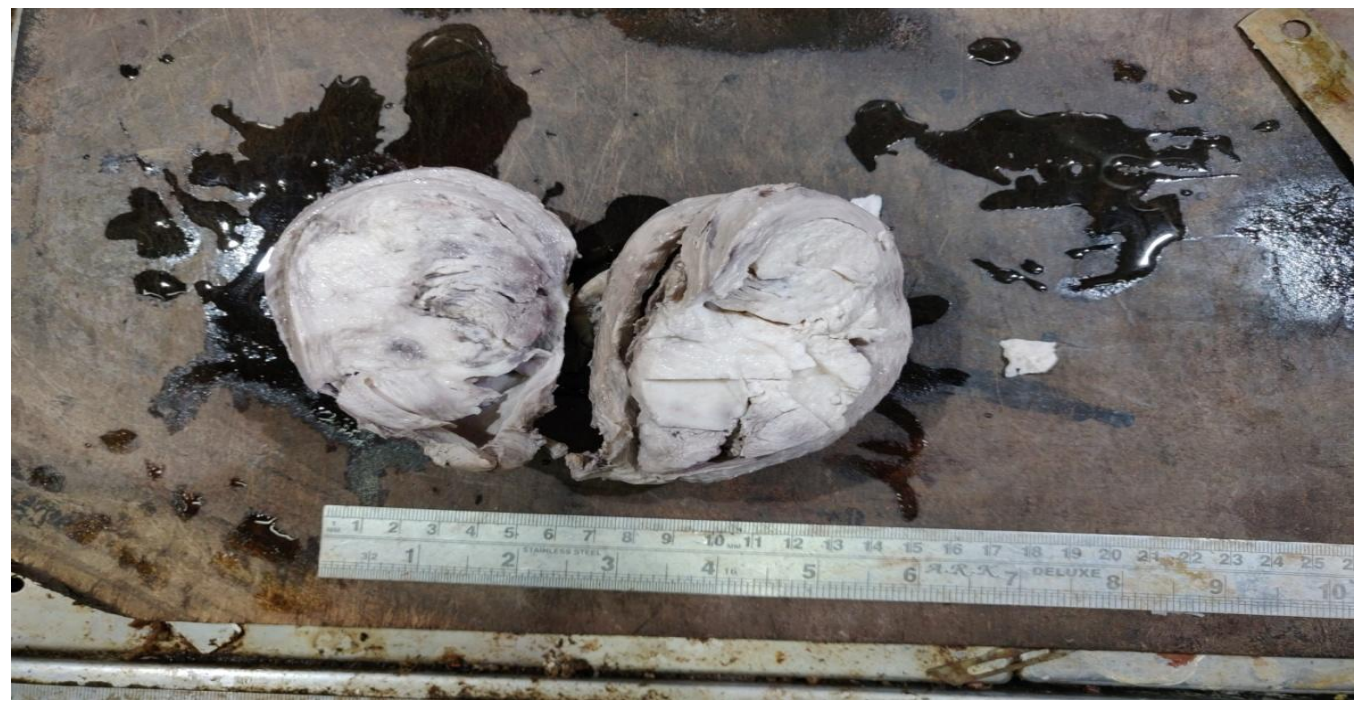

\section{Polypoid mass in uterine cavity}

\section{Microscopic examination}

Sections from the tumor showed biphasic pattern. Glandular component was showing appearance of papillary endometroid adenocarcinoma. Sarcomatous component consisted of heterologous variety having component of leiomyosarcoma, osteosarcoma and chondrosarcoma . Tumor was invading the more than half of the myometrium. Focal scattered atypical cells were also seen on the serosal surface at places. Cervix and bilateral adnexa were free of tumor. Lymphovascular invasion not seen.

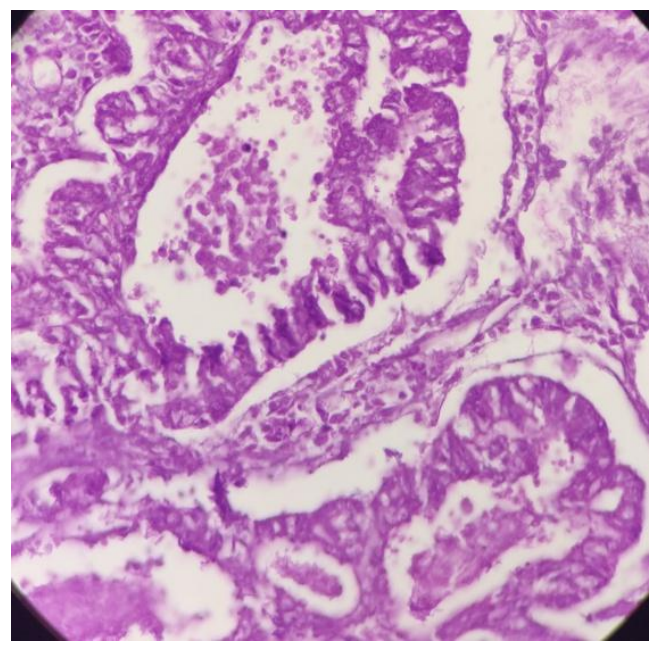

Carcinomatous component

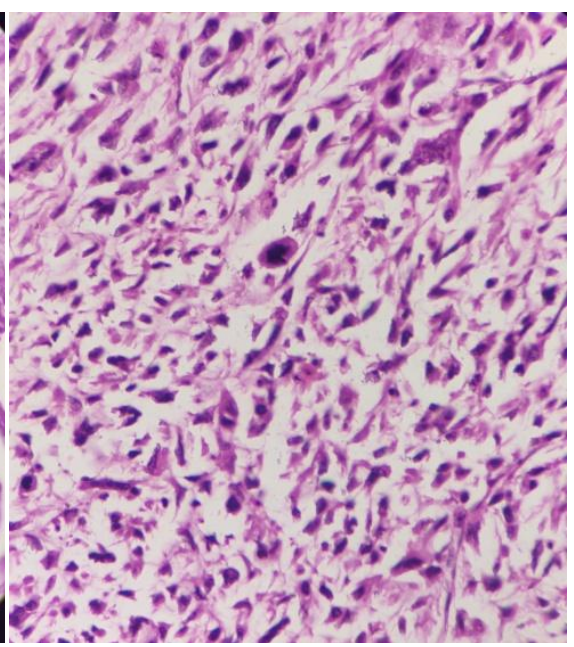

Sarcomatous component 


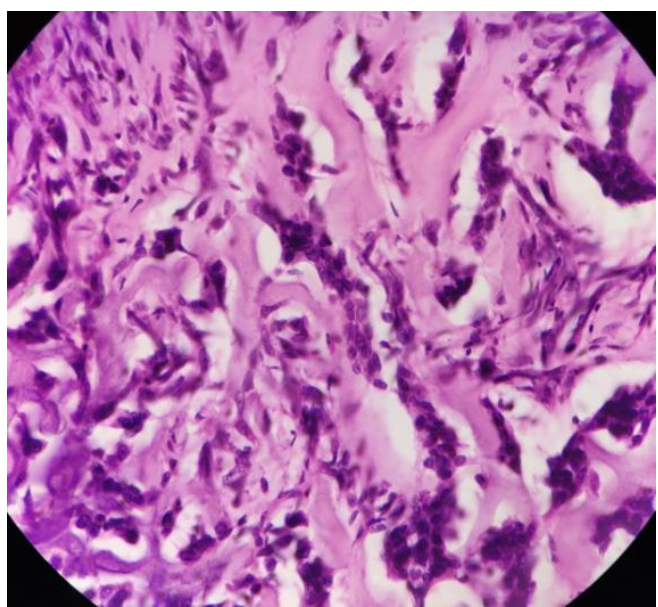

Osteosarcomatous component

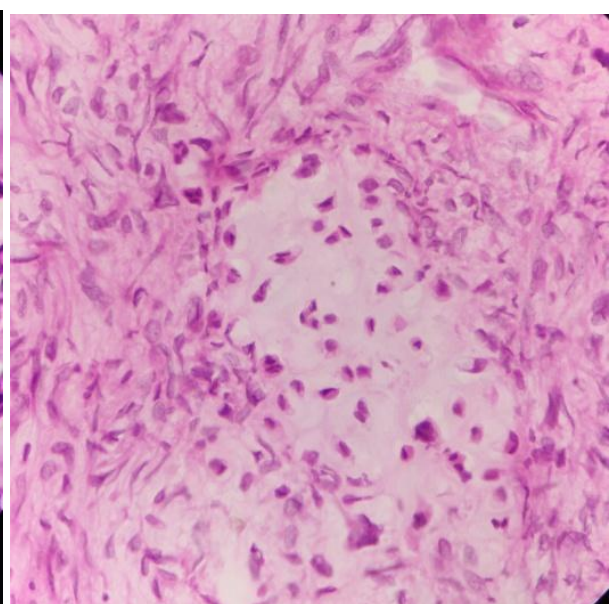

Chondrosarcomatous component

\section{Discussion:-}

Malignant uterine neoplasms containing both carcinomatous and sarcomatous element are designated in WHO classification of uterine neoplasm as carcinosarcomas ${ }^{[1]}$. It representing less than $5 \%$ of all uterine tumors account for $16.4 \%$ of all death caused by a uterine malignancy ${ }^{[6]}$. Usually occur in uterus, vagina, cervix, ovary, fallopian tubes with decreasing order of frequency ${ }^{[2,3]}$. On rare occasions the female peritoneum can developed MMMT. Risk factor for the development of carcinosarcoma are similar to endometrial carcinoma including nulliparity, advanced age, obesity, radiation exposure, exogenous estrogens exposure, long term use of tamoxifen and HPV infection ${ }^{[4,5]}$. More common in non-white women than white women ${ }^{[7]}$. In Endometrial biopsy diagnosis may be missed due to small amount of tissue. This leads to misdiagnosis and mismanagement ${ }^{[8]}$. In our case also the diagnosis was missed in endometrial biopsy. Carcinosarcoma are composed of two histological subtype which are classified based on the appearance of sarcomatous component .The sarcoma of homologous type may be composed fibrosarcoma or leiomyosarcomas whereas heterologous type has mixed component including rhabdomyosarcoma, chondrosarcoma, osteosarcoma or liposarcoma ${ }^{[1]}$. In our case carcinosarcoma was heterologous type, sarcoma consisting of bone, cartilage and smooth muscle and skeletal muscle component .

It is currently believed that carcinosarcomas have a monoclonal origin from a common multidirectional progenitor stem cell. IHC study shows both the sarcomatous and carcinomatous component, often co-expression of cytokeratin and vimentin ${ }^{[9]}$.

While hysterectomy with bilateral salpingo-oopherectomy remain the mainstay of treatment , high rate of recurrence and metastasis suggest a need for lymphadenectomy and post operative adjuvant treatment ${ }^{[1]}$.

\section{Conclusion:-}

Malignant mixed mullerian tumors are rare and aggressive tumor. Clinician should have a high suspicion for MMT in postmenopausal patients presenting with vaginal discharge and USG suggesting fibroid with seconday changes. The outcome depends on the stage of disease and depth of invasion .Combination of chemotherapy and radical surgery remain the main stay of treatment.

Being rare malignant tumor and misdiagnosed in USG and D\&C the case is being presented

\section{References:-}

1. S. A. El-Nashar and A. Mariani, -Uterine carcinosarcoma, $\|$ Clinical Obstetrics and Gynecology, vol. 54, 2, pp. 292-304, 2011.

2. A. Ahuja, R. Safaya, G. Prakash, L. Kumar, and N. K. Shukla, -Primary mixed mullerian tumor of the vagina-a case report with review of the literature, $\|$ Pathology Research and Practice, vol. 207, no. 4, pp. 253255, 2011.

3. N. K. Sharma, J. I. Sorosky, D. Bender, M. S. Fletcher, and A. K. Sood, - Malignant mixed mullerian tumor (MMMT) of the cervix,ll Gynecologic Oncology, vol. 97, no. 2, pp. 442-445, 2005. 
4. R. A. de Jong, H. W. Nijman, T. F. Wijbrandi, A. K. Reyners, H. M. Boezen, and H. Hollema, "Molecular markers and clinical behavior of uterine carcinosarcomas: focus on the epithelial tumor component," Modern Pathology.

5. P. Arora, S. Rao, N. Khurana, D. Talwar, and R. Tanwar, "Malignant mixed Mullerian tumor of broad ligament with synchronous ovarian and endometrial carcinoma: a rare association," Journal of Cancer Research and Therapeutics, vol. 7, no. 1, pp. 88-91, 2011.

6. J. S. Bosquet, S. A. Terstriep, W. A. Cliby et al., - The impact of multi-modal therapy on survival for uterine carcinosarcomas,\| Gynecologic Oncology, vol. 116, no. 3, pp. 419-423, 2010.

7. Brooks SE, Zhan M, Cote T, et al. Surveillance, epidemiology, and end results analysis of 2677 cases of uterine sarcoma 1989-1999. Gynecol Oncol. 2004;93:204-208.

8. Afonso JF. Mixed mesodermal tumors of the uterus. West J Med. 1974;120(1):17-26.

9. George E, Manivel JC, Dehner LP, et al. Malignant mixed Mullerian tumors: an immunohistochemical study of 47 cases, with histogenetic considerations and clinical correlation. Hum Pathol 1991;22:215-23. 